\title{
The feeding habits of co-occurring cod and haddock larvae from Georges Bank*
}

\author{
Joseph Kane \\ US Department of Commerce, National Oceanic and Atmospheric Administration, National Marine Fisheries Service, \\ Northeast Fisheries Center, Narragansett Laboratory, Narragansett, Rhode Island 02882, USA
}

\begin{abstract}
Types, number, size, biomass, and selection of food items by the larvae of Atlantic cod Gadus morhua and haddock Melanogrammus aeglefinus were determined by examination of gut contents from co-occurring larvae captured in plankton samples from Georges Bank. Eggs, nauplii, and copepodite stages of copepods were the predominant food items for both larvae, but cod consumed larger prey at an earlier age than haddock. The smallest larvae were the most euryphagous, haddock more than cod. Cod and haddock feeding intensity reached a peak shortly before sunset, and both larvae selected against the copepodite stages of Oithona similis. Dietary niche breadth and overlap indices indicate that competition is severe between and among similar sized individuals of both species.
\end{abstract}

\section{INTRODUCTION}

The larvae of 2 important commercial fish species, Atlantic cod Gadus morhua and haddock Melanogrammus aeglefinus often co-occur in the Northwest Atlantic Ocean. Field studies indicate that these closely related species have similar feeding habits (Marak, 1960; Sherman et al., 1981), and laboratory studies by Laurence $(19 ; 8)$ show that differences in their growth, respiration, and delayed feeding abilities are not significant. However, for unknown reasons, when the larvae were raised together in aquaria, haddock mortality was substantially higher and they grew more slowly than cod (Laurence et al., 1981).

High mortality has been observed frequently when co-occurring species were raised together in the laboratory (Krebs, 1972). Theoretically, the coexistence of these species in natural communities can be explained if: (1) competition does not exist because of unlimited resources, or (2) competition has influenced evolutionary development causing one species to occupy a different feeding niche. Since starvation during the larval stage is believed to be a key factor determining yearly fluctuations in recruitment success (Hunter, 1976), it is important to understand the competitive feeding interactions between cod and haddock larvae.

\footnotetext{
- MARMAP Contribution No. MED/NEFC 82-38
}

During the spring of 1980 large numbers of cooccurring cod and haddock larvae were collected on Georges Bank. The feeding habits of both species were analysed and compared for differences in diet composition, feeding periodicity, prey-size selection, and prey selectivity. Dietary niche breadth and overlap indices were used to evaluate competition between and within species. In addition, morphological measurements were made in an effort to discern if either species has a competitive advantage due to differential development. It is the purpose of this paper to characterize better the feeding ecology of larval cod and haddock and to determine which of the 2 principles, stated above, govern interactions during their early life history.

\section{MATERIALS AND METHODS}

Larval fish were sorted from plankton samples collected in late spring aboard the USSR research vessel 'Evrika' on Georges Bank (Fig. 1) by the National Marine Fisheries Service, Northeast Fisheries Center, in conjunction with the Marine Resources Monitoring Assessment and Prediction (MARMAP) program (Sherman, 1980). Five series of samples were taken over a $30 \mathrm{~h}$ period at approximately 1900,2400, 0800, 1200 and again at $1900 \mathrm{~h}$ EST. A $61 \mathrm{~cm}$ bongo (Posgay and Marak, 1980) equipped with an opening-closing device and a $0.333 \mathrm{~mm}$ mesh net, was towed at 1.5 


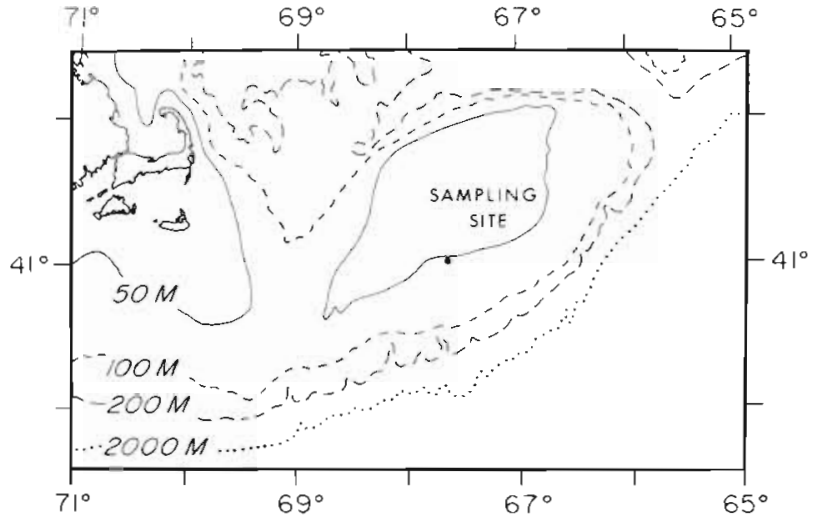

Fig. 1. Location of sampling site on Georges Bank, a portion of the US continental shelf, east of Cape Cod, Massachusetts

knots for 5 min at a discrete depth. The microzooplankton community was sampled $48 \mathrm{~h}$ earlier using a submersible pump lowered to discrete depths for $10 \mathrm{~min}$. Water was pumped on board and filtered through a $0.053 \mathrm{~mm}$ mesh net. Desired sampling depths for both pump and bongo were 5, 15, 25 and $35 \mathrm{~m}$, but varied according to wire angle. A bathykymograph was attached above the bongo and pump to measure actual sampling depth. Samples were preserved in $5 \%$ Formalin*.

In the laboratory, the entire digestive tract was dissected from each specimen and opened using mounted surgical needles under a dissecting microscope. All prey items were identified to species and life stage except for the nauplii of Pseudocalanus minutus and Calanus finmarchicus, which were grouped together (calanoid nauplii) because the digestive process often distorted key taxonomic characteristics. Standard length, skull width, eye height, and maxillary length were recorded for each larva. For the initial series of larvae analysed, a subsample of 30 specimens of each species was examined from each depth. It was later judged that 10 specimens from each depth provided sufficient information since feeding patterns were uniform throughout the water column.

Biomass of predominant food items was estimated using the width-wet weight conversion equation given by Pearre (1980). Width measurements were determined from organisms collected during the cruise by means of either Niskin bottle or pump. Since information on the weight of copepod eggs is not available, they were assigned wet weight values equivalent to the Stage III nauplius because calanoid nauplii are believed to live on the stored food of the egg until this stage is reached (Marshall and Orr, 1955).

\footnotetext{
- Reference to trade names does not imply endorsement by the National Marine Fisheries Service, NOAA
}

The percentage composition of potentially available food was determined by the sorting and identification. of organisms from both bongo and pump samples. Only counts of large zooplankters (width $>0.3 \mathrm{~mm}$ ) were used from bongo tows because the food of larval fish is not fully retained by $0.333 \mathrm{~mm}$ mesh nets (Colton et al., 1980). Though pump samples were collected $48 \mathrm{~h}$ prior to bongo samples, it was unlikely that the microzooplankton community had changed significantly since counts of large copepods from both sampling devices were comparable and weather was calm in the intervening hours. Nonetheless, in order to reduce bias caused by the sampling discrepancy, abundance data from different depths and sampling times were combined and averaged. Larvae were also combined regardless of time or depth of capture for comparisons between ingested and available food. Certain items in the resource base were omitted from prey abundance estimates because they were judged to be unavailable' to the larvae as food due to size or external morphology.

Numerical percentages of food items were adjusted to account for their frequency occurrence because percentages alone may be misleading indicators of selectivity (Berg, 1979). For example, it is possible that a food item may occur in only a few specimens but still comprise a large portion of the total number of prey. Using the strategy outlined by Crecco and Blake (1982), numerical percentages were adjusted to account for frequency occurrence as follows:

$$
\text { A } \% N_{i}=\left[\left(\% N_{i} \cdot F_{i}\right) / \sum_{i}^{W} \% N_{i} \cdot F_{i}\right] \cdot 100
$$

where $A \% \mathrm{~N}_{\mathrm{i}}=$ adjusted $\%$ composition of prey $i_{\text {; }}$ $\% N_{i}=\%$ composition of prey $i ; F_{i}=$ frequency by occurrence of prey $i ; w=$ total number of prey.

Selective feeding was examined by comparing each resource item one at a time using the Shorigin Index (Shorigin, 1939) as modified by Berg (1979):

$$
\mathrm{SEL}=\log \frac{\mathrm{A} \% \mathrm{~N}_{\mathrm{i}} \text { in the ingested food }}{\% \mathrm{~N}_{\mathrm{i}} \text { in available food }}
$$

A selectivity value (SEL) of 0 indicates random feeding on the resource item.

Dietary niche breadth and niche overlap indices were calculated in order to compare the utilization of food items to the entire spectrum of resources available. Niche breadth is defined here as the degree to which the larvae utilize available food items in proportion to their abundance in the environment. A widely used breadth index is the Proportional Similiarity Index (PS), most recently given by Feinsinger et al. (1981):

$$
\text { PS }=1-0.5 \sum_{i}^{W} \mid A \% N_{1}-\% N_{i} \text { in available food } \mid
$$


A 0 value is obtained when the predator is feeding exclusively on the rarest prey in the environment. PS approaches 1 when the feeding is totally random. Colwell and Futuyma (1971) modified the PS index to compare dietary niche overlap between and within species:

$$
\mathrm{PS}=1-0.5 \sum_{\mathrm{i}}^{\mathrm{W}}\left|\mathrm{A} \% \mathrm{~N}_{\mathrm{ic}}-\mathrm{A} \% \mathrm{C}_{1 \mathrm{~h}}\right|
$$

where $A \% N_{1 C}-A \% C_{i h}=$ adjusted $\%$ composition of prey $\mathrm{i}$ in the diet of cod (c) and haddock (h) larvae. Here, PS will approach 1 when resources are shared equally and 0 when diets are entirely different.

Larvae were subdivided into 4 size groups in order to assess changes in feeding habits with growth. The length intervals chosen reflect the size distribution of the larvae collected, rather than any distinct changes occurring in the larvae with age. The term larvae is defined here as all fish $10.0 \mathrm{~mm}$ or less, though Formalin preservation may have caused shrinkage.

\section{RESULTS}

\section{Zooplankton community}

A total of 23 zooplankton taxa were identified in the sampling area (Table 1). Copepod species were the most abundant components, comprising $64.25 \%$ of the total community. Oithona similis was the dominant copepod. Polychaete and Lamellibranch larvae constituted the bulk of non-copepod forms.

\section{Diet composition}

Qualitative and quantitative results of the analysis are presented in Tables 2-5. For both cod and haddock, feeding incidence was nearly $100 \%$ and feeding behavior was consistent throughout the water column. The predominant components of the larval diet were the different life stages of Pseudocalanus minutus, Oithona similis, Calanus finmarchicus, and Centropages typicus. In addition, phytoplankton remains were often found in the tracts of smaller larvae as a green 'slurry'. Due to their fragile nature, it was impossible to identify positively and count individual phytoplankters; thus their contribution (numbers and biomass) to the diet could not be determined. The only exception was the armoured dinoflagellate Peridinium sp. which remained intact throughout the gut, but it is probably of no nutritive value since it resists digestion. Lamellibranch larvae were omitted from the biomass analysis because they also were found intact throughout the digestive tract.
Table 1. Percentage composition of zooplankton community

\begin{tabular}{|c|c|}
\hline & $\%$ of total \\
\hline \multicolumn{2}{|l|}{ Copepods } \\
\hline Oithona similis, adults & 1.34 \\
\hline Oithona similis, copepodites (C) & 5.28 \\
\hline Oithona similis, V \& VI Nauplii (N) & 8.94 \\
\hline Oithona similis, III \& IV N & 5.71 \\
\hline Oithona similis, I \& II N & 4.38 \\
\hline Pseudocalanus minutus, adults & 2.09 \\
\hline Pseudocalanus minutus, V C & 0.38 \\
\hline Pseudocalanus minutus, III \& IV C & 0.40 \\
\hline Pseudocalanus minutus, I \& II C & 0.28 \\
\hline Calanus finnarchicus, adult & 0.49 \\
\hline Calanus finmarchicus, V C & 2.11 \\
\hline Calanus finmarchicus, IV C & 1.88 \\
\hline Calanus finmarchicus, III C & 0.28 \\
\hline Calanus finmarchicus, II C & 0.17 \\
\hline Calanus finmarchicus, I C & 0.47 \\
\hline Paracalanus parrus, adults & 0.01 \\
\hline Paracalanus parvus, V C & 0.63 \\
\hline Calanoid nauplii, $>0.35 \mathrm{~mm}$ & 1.47 \\
\hline Calanoid nauplii, $\quad 0.25-0.35 \mathrm{~mm}$ & 5.09 \\
\hline Calanoid nauplii, $<0.25 \mathrm{~mm}$ & 2.66 \\
\hline Centropages typicus, adults & 0.36 \\
\hline Centropages typicus, copepodites & 0.99 \\
\hline Centropages typicus, nauplii & 3.53 \\
\hline Temora longicornis, copepodites & 0.02 \\
\hline Temora longicornis, nauplii & 0.09 \\
\hline Microsetella spp., copepodites & 0.21 \\
\hline Microsetella spp., nauplii & 0.01 \\
\hline Acartia spp., copepodites & 0.03 \\
\hline Acartia spp., nauplii & 0.16 \\
\hline Harpacticoid, nauplii & 0.18 \\
\hline Oncaea spp., copepodites & 0.04 \\
\hline \multicolumn{2}{|l|}{ Copepod eggs } \\
\hline P. minutus \& C. finmarchicus & 10.8 \\
\hline O. similis (\# of clusters) & 0.76 \\
\hline P. parvus \& C. typicus & 2.95 \\
\hline \multicolumn{2}{|l|}{ Other invertebrates } \\
\hline Polychaete larvae & 19.43 \\
\hline Lamellibranch larvae & 9.48 \\
\hline Echinoderm larvae & 2.76 \\
\hline Chaetognaths & 1.38 \\
\hline Chaetognath eggs & 0.88 \\
\hline Unidentified eggs & 0.92 \\
\hline Gastropod larvae & 0.43 \\
\hline Decapod larvae & 0.11 \\
\hline Balanidae (cypris stage) & 0.20 \\
\hline Ostracoda & 0.02 \\
\hline Thysanoessa raschii & 0.07 \\
\hline Hyperidae & 0.03 \\
\hline Gammaridae & 0.04 \\
\hline
\end{tabular}

Copepod eggs were the most abundant food item consumed by both larvae (Tables 1 and 2). Their dietary contribution may have been slightly overestimated since intact eggs were occasionally found in the hindgut, but the majority of eggs (ca. $90 \%$ ) were in various stages of digestion throughout the gut. It should be noted that the pelagic eggs of Calanus finmarchicus are actively preyed upon by the larvae while those of 
Pseudocalanus minutus are coincidental with ingested ovigerous females who retain their eggs until hatched (Corkett and McLaren, 1978).

Both species were feeding on the same components of the prey assemblage, but substantial differences in numbers and biomass of food items consumed were found between the 2 smaller size groupings. For larvae less than $5.0 \mathrm{~mm}$, mean food item biomass was 2.5 times greater for cod than for haddock (Tables 4 and 5). This discrepancy is primarily due to the presence of Pseudocalanus minutus copepodites and adults in the diet of cod and their virtual absence in haddock (Fig. 2). In the next larger size grouping, 5.0 to $5.9 \mathrm{~mm}$, haddock begin to utilize larger prey items, but in numbers far below those which cod ingest. For exam- ple, adult $P$. minutus females were found in $52 \%$ of the cod examined compared to $15 \%$ of the haddock. Consequently, mean biomass per cod larva (0.1574 mg) was nearly double that of haddock $(0.0796 \mathrm{mg})$. Haddock larvae 6.0 to $6.9 \mathrm{~mm}$ began to compete with cod for larger prey; both species fed heavily on $P$. minutus adult females. Diets of both larvae in the 7.0 to $10.0 \mathrm{~mm}$ size category were nearly identical.

The differences in ingested biomass between the younger larvae may not be as large as indicated if phytoplankton has nutritive value. Haddock were more omnivorous than cod. Phytoplankton 'slurry' was present in $78 \%$ of all haddock under $5.0 \mathrm{~mm}$, in contrast to only $39 \%$ of the cod examined. Furthermore, though not digestible, Peridinians were found in $31 \%$

Table 2. Types and frequency occurrence of food items found in larval haddock. Specimens collected at different times and from discrete depths have been combined

\begin{tabular}{|c|c|c|c|c|c|c|c|c|c|c|}
\hline \multirow{2}{*}{$\begin{array}{c}\text { Size group: } \\
\text { No. larvae examined: } \\
\text { Prey items }\end{array}$} & \multicolumn{2}{|c|}{$\begin{array}{c}3.5-4.9 \mathrm{~mm} \\
36\end{array}$} & \multicolumn{2}{|c|}{$\begin{array}{c}5.0-5.9 \mathrm{~mm} \\
96\end{array}$} & \multicolumn{2}{|c|}{$\begin{array}{c}6.0-6.9 \mathrm{~mm} \\
80\end{array}$} & \multicolumn{2}{|c|}{$\begin{array}{c}7.0-10.0 \mathrm{~mm} \\
52\end{array}$} & \multicolumn{2}{|c|}{$\begin{array}{c}\text { All larvae } \\
264\end{array}$} \\
\hline & No. & Freq. & No. & Freq. & No. & Freq. & No. & Freq. & No. & Freq. \\
\hline \multicolumn{11}{|l|}{ Copepod eggs } \\
\hline Calanus finmarchicus & 531 & 89 & 619 & 95 & 373 & 73 & 200 & 62 & 1345 & 81 \\
\hline Pseudocalanus minutus & - & - & 42 & 2 & 388 & 19 & 462 & 33 & 892 & 13 \\
\hline Oithona similis & - & - & - & - & - & - & 1 & 2 & 1 & $<1$ \\
\hline \multicolumn{11}{|l|}{ Calanoid nauplii (N) } \\
\hline $0.25-35 \mathrm{~mm}$ lenth & 65 & 69 & 334 & 74 & 227 & 74 & 64 & 39 & 690 & 66 \\
\hline$>0.35 \mathrm{~mm}$ length & 8 & 19 & 45 & 32 & 42 & 31 & 13 & 17 & 108 & 27 \\
\hline \multirow{2}{*}{\multicolumn{11}{|c|}{ Pseudocalanus minutus }} \\
\hline & - & - & 18 & 15 & 70 & 56 & 130 & 89 & 218 & 40 \\
\hline adult males & - & - & - & - & 7 & 9 & 11 & 21 & 18 & 7 \\
\hline$V$ copepodites & - & - & 9 & 7 & 16 & 19 & 27 & 40 & 52 & 16 \\
\hline III \& IV copepodites & - & - & 18 & 17 & 34 & 30 & 27 & 33 & 79 & 22 \\
\hline I \& II copepodites & 2 & 6 & 16 & 15 & 30 & 33 & 10 & 15 & 58 & 19 \\
\hline \multicolumn{11}{|l|}{ Oithona similis } \\
\hline aduits & 2 & 3 & 7 & 7 & 15 & 15 & 12 & 20 & 36 & 11 \\
\hline copepodites & 6 & 17 & 20 & 19 & 16 & 19 & 4 & 8 & 46 & 16 \\
\hline $\mathrm{V} \& \mathrm{VIN}$ & 11 & 22 & 69 & 49 & 38 & 24 & 6 & 10 & 124 & 30 \\
\hline III \& IV N & 14 & 25 & 29 & 22 & 14 & 13 & 2 & 2 & 59 & 16 \\
\hline \multicolumn{11}{|l|}{ Centropages typicus } \\
\hline copepodites & - & - & 4 & 4 & 6 & 6 & 4 & 4 & 14 & 4 \\
\hline VI N & 2 & 6 & 6 & 6 & 3 & 4 & 1 & 2 & 12 & 5 \\
\hline IV \& V N & 5 & 11 & 28 & 18 & 4 & 4 & 1 & 2 & 38 & 10 \\
\hline II \& III N & 2 & 6 & 1 & 1 & 2 & 3 & - & - & 5 & 2 \\
\hline Paracalanus parvus, adults & 1 & 3 & 1 & 1 & - & - & - & - & 2 & $<1$ \\
\hline Acartia spp., nauplii & - & - & 3 & 3 & - & - & - & - & 3 & 1 \\
\hline Centropages hamatus, adults & - & - & - & - & - & - & 1 & 2 & 1 & $<1$ \\
\hline Lamellibranch, larvae & 24 & 11 & 45 & 18 & 7 & 6 & - & - & 76 & 10 \\
\hline Chaetognath, eggs & - & - & 3 & 1 & - & - & - & - & 3 & $<1$ \\
\hline \multicolumn{11}{|l|}{ Phytoplankton } \\
\hline 'Slurry' & 28 & 78 & 31 & 32 & 2 & 3 & 1 & 2 & 62 & 23 \\
\hline Peridinium spp. & 30 & 36 & 128 & 48 & 32 & 24 & 4 & 6 & 194 & 31 \\
\hline Ceratium spp. & - & - & 1 & 1 & 2 & 3 & - & - & 3 & 1 \\
\hline Total no. prey & 353 & & 1477 & & 1328 & & 981 & & 4139 & \\
\hline Mean no. prey larva ${ }^{-1}$ & 10 & & 15 & & 17 & & 19 & & 16 & \\
\hline No. empty & - & & 1 & & - & & - & & 1 & \\
\hline
\end{tabular}


of all haddock analysed while cod completely avoided the heavily armoured phytoplankter.

\section{Prey size}

Results of all larval fish feeding studies have indicated that as larvae increase in size there is a corresponding increase in the amount of larger prey ingested. The present study was no exception; mean prey width increased with length of larvae (Fig. 3), though the increase was negligible between the 2 smallest haddock length intervals. The histogram readily shows that cod larvae from Georges Bank feeds on larger prey than haddock.

There was a great demand by both species for food with widths between 0.10 and $0.19 \mathrm{~mm}$ (Fig. 4). Smal- ler items are selected, but they are gradually eliminated with growth of larvae. One would expect a greater utilization of prey between 0.20 and $0.29 \mathrm{~mm}$ during growth, but they were not abundant in the water column. Fig. 3 also reflects the 2 different feeding patterns of the larvae. If the biomass contribution of each prey group was illustrated instead of numbers, the differences between the 2 species would be widened considerably.

\section{Prey selectivity}

Larval cod, and presumably haddock, locate their prey by sight and draw the food item into their mouth by rapid expansion of the buccal cavity (Ellertsen et al., 1980). Therefore, size, visibility, evasion speed, and

Table 3. Types and frequency occurrence of food items found in larval cod. Specimens collected at different times and from discrete depths have been combined

\begin{tabular}{|c|c|c|c|c|c|c|c|c|c|c|}
\hline \multirow{2}{*}{$\begin{array}{c}\text { Size group: } \\
\text { No. larvae examined: } \\
\text { Prey items }\end{array}$} & \multicolumn{2}{|c|}{$\begin{array}{c}3.5-4.9 \mathrm{~mm} \\
28\end{array}$} & \multicolumn{2}{|c|}{$\begin{array}{c}5.0-5.9 \mathrm{~mm} \\
62\end{array}$} & \multicolumn{2}{|c|}{$\begin{array}{c}6.0-6.9 \mathrm{~mm} \\
42\end{array}$} & \multicolumn{2}{|c|}{$\begin{array}{c}7.0-10.0 \mathrm{~mm} \\
42\end{array}$} & \multicolumn{2}{|c|}{$\begin{array}{c}\text { All larvae } \\
174\end{array}$} \\
\hline & No. & Freq. & No. & Freq. & No. & Freq. & No. & Freq. & No. & Freq. \\
\hline \multicolumn{11}{|l|}{ Copepod eggs } \\
\hline Calanus finmarchicus & 87 & 89 & 233 & 76 & 119 & 43 & 83 & 45 & 522 & 63 \\
\hline Pseudocalanus minutus & - & - & 92 & 8 & 185 & 14 & 282 & 29 & 559 & 13 \\
\hline \multicolumn{11}{|l|}{ Calanoid nauplii (N) } \\
\hline $0.25-35 \mathrm{~mm}$ lenth & 75 & 79 & 217 & 77 & 103 & 81 & 30 & 36 & 425 & 68 \\
\hline$>0.35 \mathrm{~mm}$ length & 8 & 25 & 26 & 31 & 18 & 21 & 5 & 12 & 57 & 23 \\
\hline \multicolumn{11}{|l|}{ Pseudocalanus minutus } \\
\hline adult females & 5 & 18 & 40 & 52 & 55 & 64 & 100 & 88 & 200 & 58 \\
\hline adult males & 1 & 4 & 3 & 5 & 6 & 14 & 9 & 19 & 19 & 10 \\
\hline$V$ copepodites & 4 & 14 & 23 & 31 & 22 & 38 & 28 & 50 & 77 & 35 \\
\hline III \& IV copepodites & 6 & 14 & 26 & 34 & 37 & 55 & 22 & 43 & 91 & 38 \\
\hline I \& II copepodites & 3 & 11 & 15 & 23 & 13 & 26 & 14 & 29 & 45 & 23 \\
\hline \multicolumn{11}{|l|}{ Oithona similis } \\
\hline adults & 2 & 7 & 6 & 10 & 8 & 14 & 11 & 19 & 27 & 13 \\
\hline copepodites & 7 & 21 & 15 & 16 & 11 & 24 & 1 & 2 & 34 & 16 \\
\hline$V \&$ VI N & 19 & 36 & 35 & 37 & 19 & 21 & 7 & 17 & 80 & 28 \\
\hline III \& IV N & 6 & 21 & 16 & 21 & 8 & 10 & 1 & 2 & 31 & 13 \\
\hline \multicolumn{11}{|l|}{ Centropages typicus } \\
\hline copepodites & - & - & - & - & - & - & 5 & 7 & 5 & 2 \\
\hline VIN & - & - & 2 & 3 & 3 & 5 & 1 & 2 & 6 & 3 \\
\hline IV \& V N & 1 & 4 & 7 & 10 & 1 & 2 & 1 & 2 & 10 & 5 \\
\hline II \& III N & 1 & 4 & 1 & 2 & - & - & - & - & 2 & 1 \\
\hline \multicolumn{11}{|l|}{ Paracalanus parvus } \\
\hline adults & - & - & 2 & 3 & - & - & - & - & 2 & 1 \\
\hline V copepodites & - & - & 1 & 2 & - & - & - & - & 1 & $<1$ \\
\hline Lamellibranch, larvae & 3 & 7 & 3 & 5 & 1 & 2 & - & - & 7 & 3 \\
\hline \multicolumn{11}{|l|}{ Phytoplankton } \\
\hline 'Slurry' & 11 & 39 & 5 & 8 & - & - & - & - & 16 & 9 \\
\hline Peridinium spp. & - & - & - & - & 1 & 2 & - & - & 1 & $<1$ \\
\hline Ceratium spp. & 2 & 7 & - & - & - & - & - & - & 2 & 1 \\
\hline Total no. prey & 241 & & 768 & & 610 & & 600 & & 2219 & \\
\hline Mean no. prey larva ${ }^{-1}$ & 9 & & 12 & & 15 & & 14 & & 13 & \\
\hline No. empty & 1 & & 1 & & - & & 1 & & 3 & \\
\hline
\end{tabular}




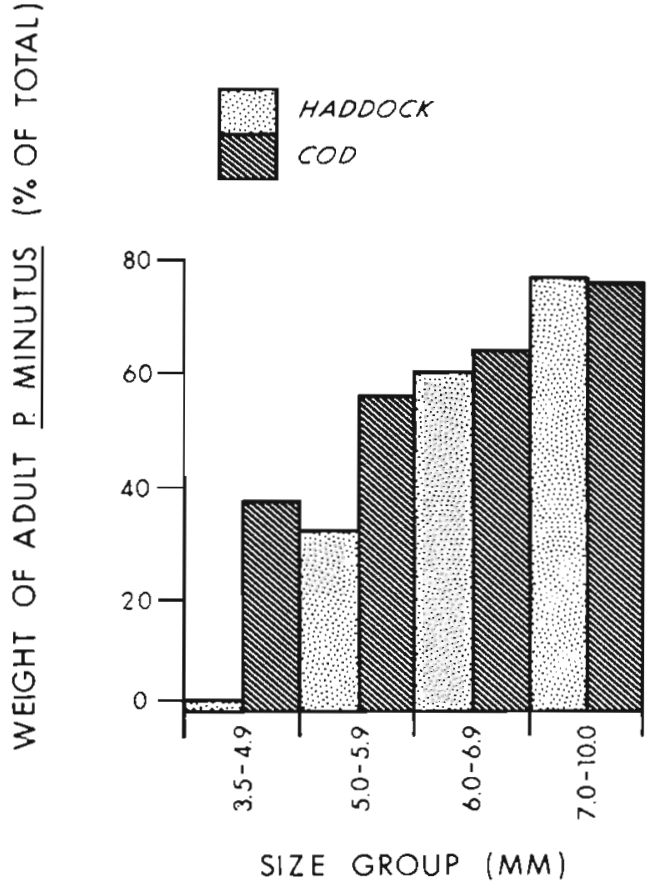

Fig. 2. Comparison of the wet weight contribution of Pseudocalanus minutus adults in the diet of cod and haddock larvae

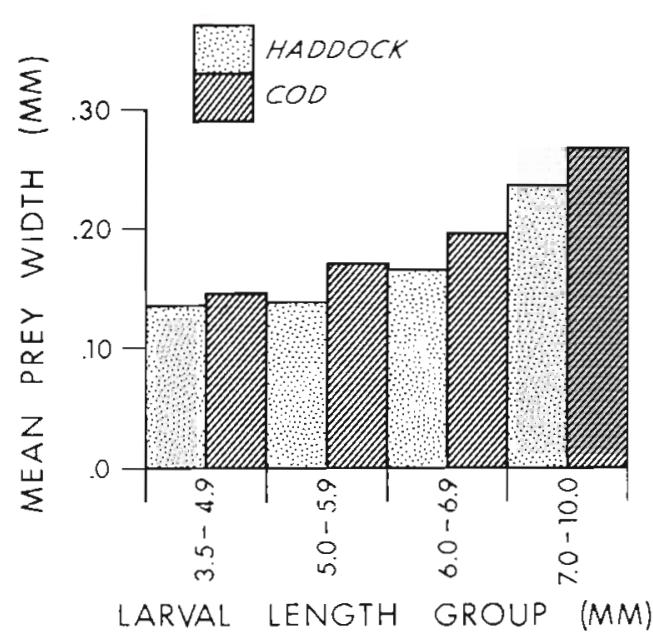

Fig. 3. Food size of haddock and cod larvae. Mean prey size increases with length, though the increase is negligible for the two smallest haddock length intervals

Table 4. Biomass of major prey items of haddock larvae (all specimens combined)

\begin{tabular}{|c|c|c|c|c|c|c|c|c|c|c|}
\hline \multirow{2}{*}{$\begin{array}{c}\text { Size group: } \\
\text { No. larvae examined: } \\
\text { Prey items }\end{array}$} & \multicolumn{2}{|c|}{$\begin{array}{c}3.5-4.9 \mathrm{~mm} \\
36\end{array}$} & \multicolumn{2}{|c|}{$\begin{array}{c}5.0-5.9 \mathrm{~mm} \\
96\end{array}$} & \multicolumn{2}{|c|}{$\begin{array}{c}6.0-6.9 \mathrm{~mm} \\
80\end{array}$} & \multicolumn{2}{|c|}{$\begin{array}{c}7.0-10.0 \mathrm{~mm} \\
52\end{array}$} & \multicolumn{2}{|c|}{$\begin{array}{l}\text { All larvae } \\
264\end{array}$} \\
\hline & $\begin{array}{l}\text { Biomass } \\
\text { (mg) }\end{array}$ & $\begin{array}{l}\% \text { of } \\
\text { total }\end{array}$ & $\begin{array}{l}\text { Biomass } \\
\text { (mg) }\end{array}$ & $\begin{array}{l}\% \text { of } \\
\text { total }\end{array}$ & $\begin{array}{c}\text { Biomass } \\
\text { (mg) }\end{array}$ & $\begin{array}{l}\% \text { of } \\
\text { total }\end{array}$ & $\begin{array}{c}\text { Biomass } \\
\text { (mg) }\end{array}$ & $\begin{array}{l}\% \text { of } \\
\text { total }\end{array}$ & $\begin{array}{c}\text { Biomass } \\
\text { (mg) }\end{array}$ & $\begin{array}{l}\% \text { of } \\
\text { total }\end{array}$ \\
\hline \multicolumn{11}{|l|}{ Copepod eggs } \\
\hline Calanus finmarchicus & 0.484 & 53.0 & 1.956 & 25.7 & 1.179 & 7.4 & 0.632 & 2.7 & 4.251 & 8.9 \\
\hline Pseudocalanus minutus & - & - & 0.111 & 1.5 & 1.024 & 6.4 & 1.220 & 5.3 & 2.355 & 4.9 \\
\hline \multicolumn{11}{|l|}{ Calanoid nauplii (N) } \\
\hline $0.25-35 \mathrm{~mm}$ length & 0.221 & 24.2 & 1.136 & 14.9 & 0.772 & 4.8 & 0.218 & $<1$ & 2.347 & 4.9 \\
\hline$>0.35 \mathrm{~mm}$ length & 0.055 & 6.0 & 0.310 & 4.1 & 0.289 & 1.8 & 0.089 & $<1$ & 0.743 & 1.6 \\
\hline \multicolumn{11}{|l|}{ Pseudocalanus minutus } \\
\hline adult females & - & - & 2.466 & 32.5 & 9.590 & 60.0 & 17.810 & 76.7 & 29.866 & 62.6 \\
\hline adult males & - & - & - & - & 0.576 & 3.6 & 0.905 & 3.9 & 1.481 & 3.1 \\
\hline V copepodites & - & - & 0.387 & 5.1 & 0.687 & 4.3 & 1.160 & 5.0 & 2.234 & 4.7 \\
\hline III + IV copepodites & - & - & 0.450 & 5.9 & 0.850 & 5.3 & 0.675 & 2.9 & 1.975 & 4.1 \\
\hline I + II copepodites & 0.024 & 2.6 & 0.191 & 2.5 & 0.358 & 2.2 & 0.119 & $<1$ & 0.692 & 1.5 \\
\hline \multicolumn{11}{|l|}{ Oithona similis } \\
\hline adults & 0.041 & 4.5 & 0.144 & 1.9 & 0.308 & 1.9 & 0.246 & 1.1 & 0.739 & 1.6 \\
\hline copepodites & 0.039 & 4.3 & 0.130 & 1.7 & 0.104 & $<1$ & 0.026 & $<1$ & 0.299 & $<1$ \\
\hline $\mathrm{V}+\mathrm{VIN}$ & 0.019 & 2.1 & 0.117 & 1.4 & 0.064 & $<1$ & 0.010 & $<1$ & 0.210 & $<1$ \\
\hline III + IV N & 0.014 & 1.5 & 0.028 & $<1$ & 0.014 & $<1$ & 0.002 & $<1$ & 0.058 & $<1$ \\
\hline \multicolumn{11}{|l|}{ Centropages typicus } \\
\hline copepodites & - & - & 0.110 & 1.4 & 0.165 & 1.0 & 0.110 & $<1$ & 0.385 & $<1$ \\
\hline VI N & 0.005 & $<1$ & 0.015 & $<1$ & 0.008 & $<1$ & 0.003 & $<1$ & 0.031 & $<1$ \\
\hline $\mathrm{IV}+\mathrm{VN}$ & 0.009 & 1.0 & 0.048 & $<1$ & 0.007 & $<1$ & 0.002 & $<1$ & 0.066 & $<1$ \\
\hline $\mathrm{II}+\operatorname{III} \mathrm{N}$ & 0.002 & $<1$ & 0.001 & $<1$ & 0.002 & $<1$ & - & - & 0.005 & $<1$ \\
\hline Total & 0.913 & & 7.600 & & 15.997 & & 23.227 & & 47.737 & \\
\hline Mean & 0.025 & & 0.079 & & 0.200 & & 0.447 & & 0.181 & \\
\hline
\end{tabular}




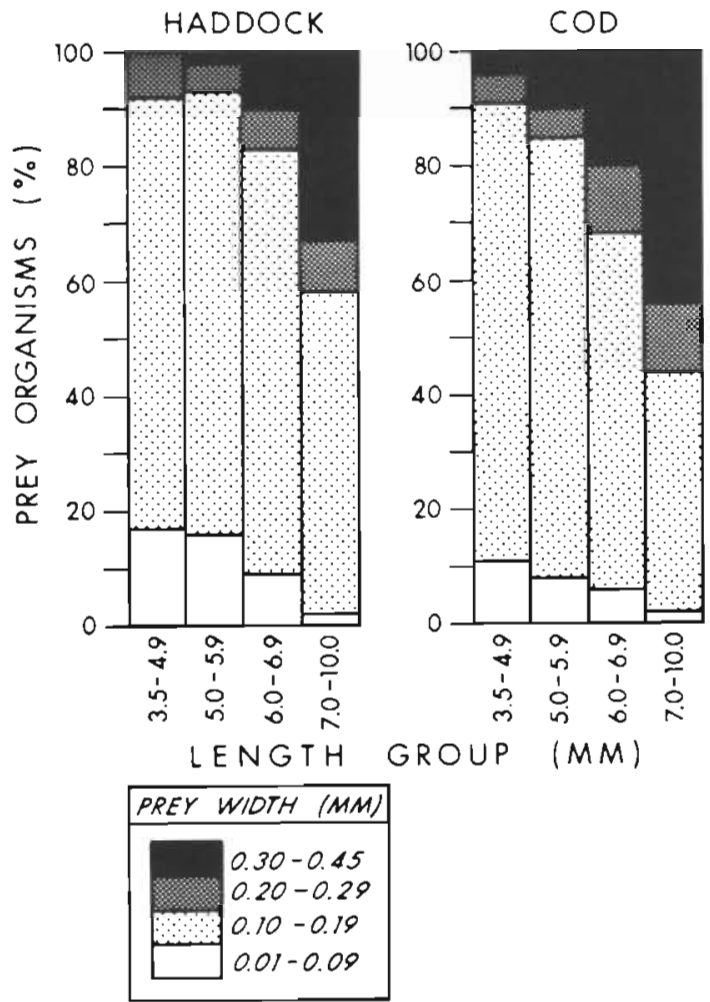

Fig. 4. Preferred prey size by larval fish length group morphological makeup of potential prey are all key factors determining prey selectivity. Size is the dominant factor and for copepods, width is the critical dimension (Blaxter, 1965; Hunter, 1981).

The largest prey consumed by both species of larvae were Pseudocalanus minutus adult females, with a mean width, excluding appendages, of $0.43 \mathrm{~mm}$ (range: 0.30 to $0.48 \mathrm{~mm}$ ). Larger copepods, such as the late stage copepodites of Calanus finmarchicus and Centropages typicus, are thus unavailable to the larvae because their size precludes them from ingestion. Small size also plays a role in selectivity. Copepod nauplii with widths less than $0.07 \mathrm{~mm}$ were virtually absent from the larval diet. Small size reduces prey vulnerability by reducing the likelihood of being sighted by visual predators, especially where prey are abundant (O'Brien, 1979).

Polychaete and Echinoderm larvae are not susceptible to larval predation due to structural adaptions. The latter are covered with chitinous setae and the former possess sharp calciferous spines, no doubt rendering them difficult and/or painful to swallow. They were major components of the zooplankton community, but absent from the larval diet.

Selectivity values of the predominant food items are given in Fig. 5 and 6. Changes in selectivity between

Table 5. Biomass of major prey items of cod larvae (all specimens combined)

\begin{tabular}{|c|c|c|c|c|c|c|c|c|c|c|}
\hline \multirow{2}{*}{$\begin{array}{c}\text { Size group: } \\
\text { No. larvae examined: } \\
\text { Prey items }\end{array}$} & \multicolumn{2}{|c|}{$\begin{array}{c}3.5-4.9 \mathrm{~mm} \\
28\end{array}$} & \multicolumn{2}{|c|}{$\begin{array}{c}5.0-5.9 \mathrm{~mm} \\
62\end{array}$} & \multicolumn{2}{|c|}{$\begin{array}{c}6.0-6.9 \mathrm{~mm} \\
42\end{array}$} & \multicolumn{2}{|c|}{$\begin{array}{c}7.0-10.0 \mathrm{~mm} \\
42\end{array}$} & \multicolumn{2}{|c|}{$\begin{array}{l}\text { All larvàe } \\
174\end{array}$} \\
\hline & $\begin{array}{c}\text { Biomass } \\
\text { (mg) }\end{array}$ & $\begin{array}{l}\% \text { of } \\
\text { total }\end{array}$ & $\begin{array}{c}\text { Biomass } \\
\text { (mg) }\end{array}$ & $\begin{array}{l}\% \text { of } \\
\text { total }\end{array}$ & $\begin{array}{c}\text { Biomass } \\
\text { (mg) }\end{array}$ & $\begin{array}{l}\% \text { of } \\
\text { total }\end{array}$ & $\begin{array}{c}\text { Biomass } \\
(\mathrm{mg})\end{array}$ & $\begin{array}{l}\% \text { of } \\
\text { total }\end{array}$ & $\begin{array}{c}\text { Biomass } \\
\text { (mg) }\end{array}$ & $\begin{array}{l}\% \text { of } \\
\text { total }\end{array}$ \\
\hline \multicolumn{11}{|l|}{ Copepod eggs } \\
\hline Calanus finmarchicus & 0.275 & 15.0 & 0.736 & 7.6 & 0.376 & 3.2 & 0.262 & 1.5 & 1.649 & 4.1 \\
\hline Pseudocalanus minutus & - & - & 0.243 & 2.5 & 0.488 & 4.2 & 0.745 & 4.2 & 1.476 & 3.6 \\
\hline \multicolumn{11}{|l|}{ Calanoid nauplii (N) } \\
\hline $0.25-35 \mathrm{~mm}$ length & 0.255 & 13.9 & 0.738 & 7.6 & 0.350 & 3.0 & 0.102 & $<1$ & 1.445 & 3.5 \\
\hline$>0.35 \mathrm{~mm}$ length & 0.055 & 3.0 & 0.179 & 1.8 & 0.124 & 1.1 & 0.034 & $<1$ & 0.392 & $<1$ \\
\hline \multicolumn{11}{|l|}{ Pseudocalanus minutus } \\
\hline adult females & 0.685 & 37.3 & 5.480 & 56.2 & 7.535 & 64.5 & 13.700 & 76.6 & 27.400 & 66.6 \\
\hline adult males & 0.082 & 4.5 & 0.247 & 2.5 & 0.494 & 4.2 & 0.741 & 4.1 & 1.564 & 3.8 \\
\hline$V$ copepodites & 0.172 & 9.4 & 0.988 & 10.1 & 0.945 & 8.1 & 1.203 & 6.7 & 3.308 & 8.0 \\
\hline III + IV copepodites & 0.150 & 8.2 & 0.650 & 6.7 & 0.925 & 7.9 & 0.550 & 3.1 & 2.275 & 5.5 \\
\hline I + II copepodites & 0.036 & 2.0 & 0.179 & 1.8 & 0.155 & 1.3 & 0.167 & $<1$ & 0.537 & 1.3 \\
\hline \multicolumn{11}{|l|}{ Oithona similis } \\
\hline adults & 0.041 & 2.2 & 0.123 & 1.3 & 0.164 & 1.4 & 0.226 & 1.3 & 0.554 & 1.3 \\
\hline copepodites & 0.046 & 2.5 & 0.096 & 1.0 & 0.072 & $<1$ & 0.007 & $<1$ & 0.221 & $<1$ \\
\hline$V+V I N$ & 0.032 & 1.8 & 0.059 & $<1$ & 0.032 & $<1$ & 0.012 & $<1$ & 0.135 & $<1$ \\
\hline III + IV N & 0.006 & $<1$ & 0.016 & $<1$ & 0.008 & $<1$ & 0.001 & $<1$ & 0.028 & $<1$ \\
\hline \multicolumn{11}{|l|}{ Centropages typicus } \\
\hline copepodites & - & - & - & - & - & - & 0.138 & $<1$ & 0.138 & $<1$ \\
\hline VI N & - & - & 0.005 & $<1$ & 0.008 & $<1$ & 0.003 & $<1$ & 0.016 & $<1$ \\
\hline $\mathrm{IV}+\mathrm{VN}$ & 0.002 & $<1$ & 0.012 & $<1$ & 0.002 & $<1$ & 0.002 & $<1$ & 0.018 & $<1$ \\
\hline $\mathrm{II}+\mathrm{III} \mathrm{N}$ & 0.001 & $<1$ & 0.001 & $<1$ & - & - & - & - & 0.002 & $<1$ \\
\hline Total & 1.838 & & 9.752 & & 11.678 & & 17.893 & & 41.160 & \\
\hline Mean & 0.066 & & 0.157 & & 0.278 & & 0.426 & & 0.237 & \\
\hline
\end{tabular}



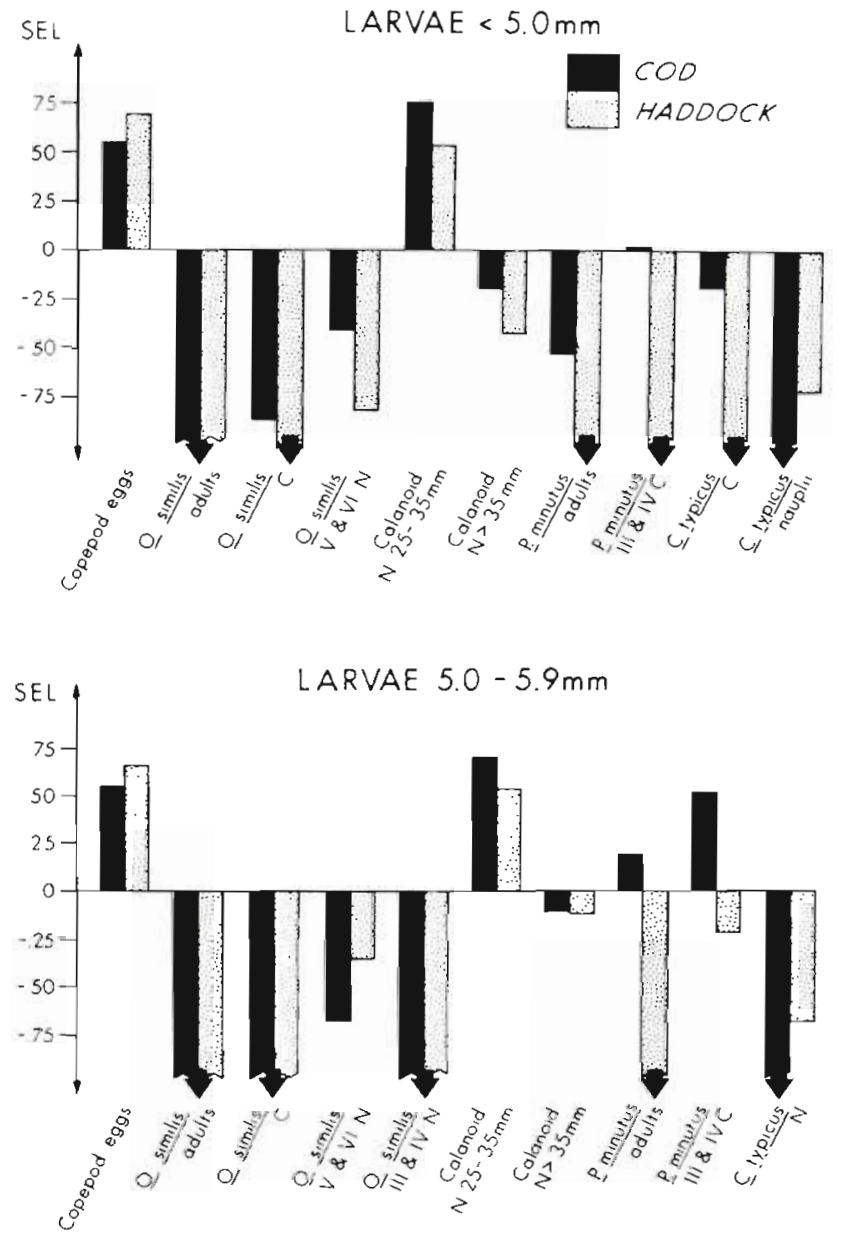

Fig. 5. Modified Shorigin indices of feeding selectivity of cod and haddock larvae $<6.0 \mathrm{~mm}$ for the predominant prey items

different size groups reflect the dominant role prey size has in influencing cod and haddock selectivity. Smaller larvae of both species strongly select for copepod eggs and calanoid nauplii. With each increasing size group, there is a greater selection for more advanced copepodite stages and adults, along with a corresponding decrease for smaller items. Copepod egg selectivity does not decrease because Pseudocalanus minutus egg consumption is increasing as larvae feed more heavily on ovigerous females. Food items below the preferred size, such as Oithona similis and Centropages typicus nauplii, are selected against by all size groups. Differences between the two species are a result of cod's early ingestion of larger prey.

All size groupings of both larvae selected against Oithona similis copepodites and adults. Rejection does not appear to be due to size, since they lie within the preferred prey size range. Cohen and Lough (1983) report that herring larvae also appear to select against $O$. similis on Georges Bank and suggest they may be unavailable because of their vertical distribution. In the samples analysed here from late May, however, $O$. similis is distributed throughout the water column.

The low incidence of Oithona similis in the diets of larval fish can be understood by examining the defenses the copepod has developed to minimize predation. Evidence in the literature indicates that $O$. similis has maximized evasion speed and has increased its width with extended antennae. Gauld (1966) reports that unlike the slow and smooth swimming form exhibited by Pseudocalanus minutus, $O$. similis moves in an erratic zig-zag pattern. Net retention studies (Saville, 1958; Colton et al., 1980) have reported that $O$. similis is retained in far greater numbers then expected on the basis of cephalothorax width because it holds its antennae perpendicular to the body instead of along the carapace, as most copepods do. The occurrence of $O$. similis in the diet increased with each larger size grouping of larvae, indicating that larger and more experienced larvae can better utilize this copepod, despite its speed and extended antennae.
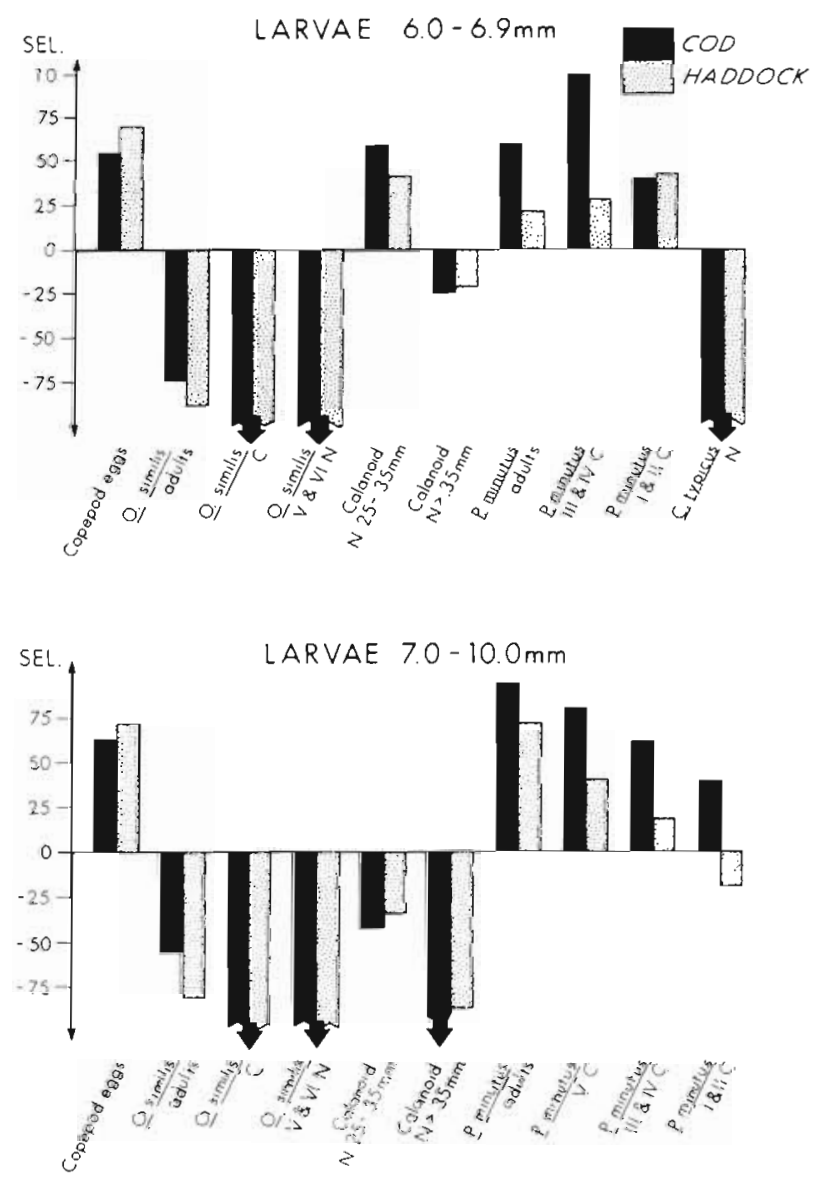

Fig. 6. Modified Shorigin indices of feeding selectivity of cod and haddock larvae $>6.0 \mathrm{~mm}$ for the predominant prey items 

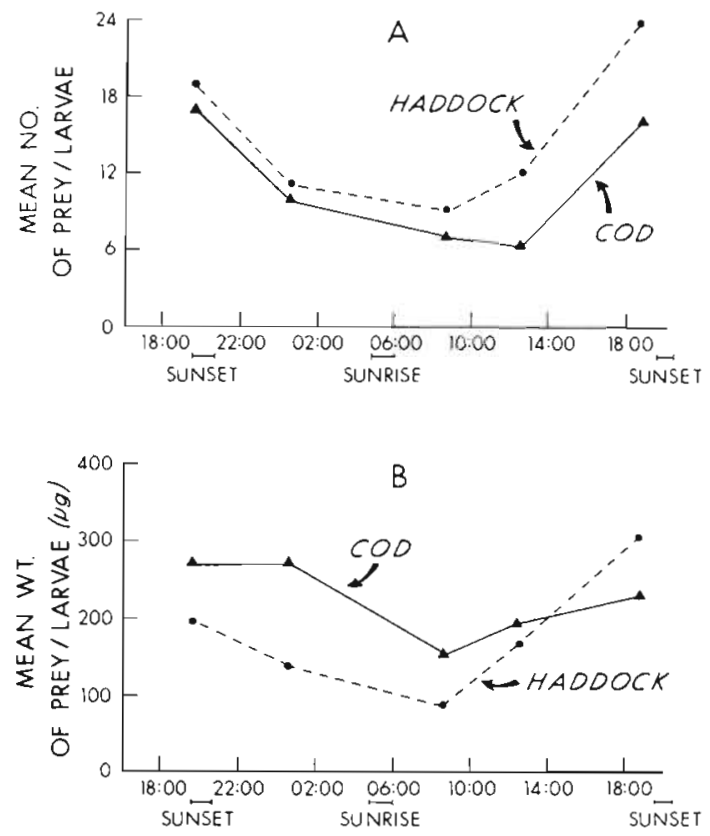

Fig. 7. Comparison of diel variations in: (A) feeding incidence, and $(B)$ mean biomass of gut contents

\section{Diel variations in feeding}

Cod and haddock larvae have similar diel feeding patterns (Fig. 7). Feeding intensity (mean no. prey larva $^{-1}$ ) reaches a peak shortly before sunset, declines steadily until after sunrise, then increases during the day. Last (1980) also found a similar pattern for North Sea cod larvae using information gathered in $2 \mathrm{~h}$ intervals. Many of the food items found in larvae collected near midnight were well digested, indicating the prey were ingested earlier, possibly at sunset. Furthermore, cod larvae, which feed on larger particles that presumably take longer to digest, did not show a decline in mean prey biomass between sunset and midnight. Since laboratory studies have shown that cod larvae are visual feeders that require a minimum light intensity for active feeding (Ellertsen et al., 1980), intact food items found in larvae collected during the night were possibly passively ingested due to high densities of prey in the water column.

Fig. 4 also demonstrates that haddock consumes more food particles than cod, but of lower biomass. This was consistent over the sampling period except at the second evening station. Here, due to the scarcity of younger haddock larvae, $67 \%$ of the specimens analysed were over $6.0 \mathrm{~mm}$. Thus, since larger larvae feed heavily on Pseudocalanus minutus adult females, many ovigerous, mean biomass and prey numbers were disproportionally higher for haddock than cod.

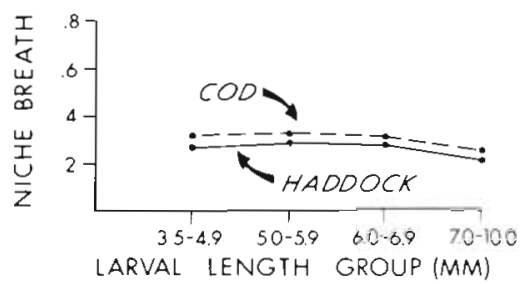

A

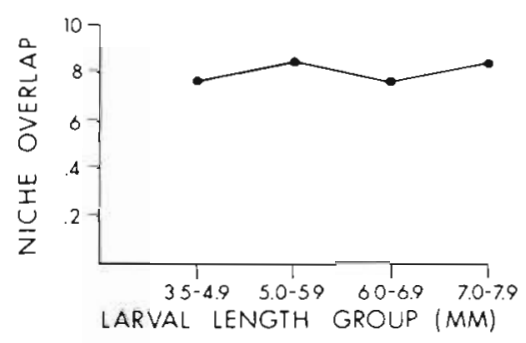

B

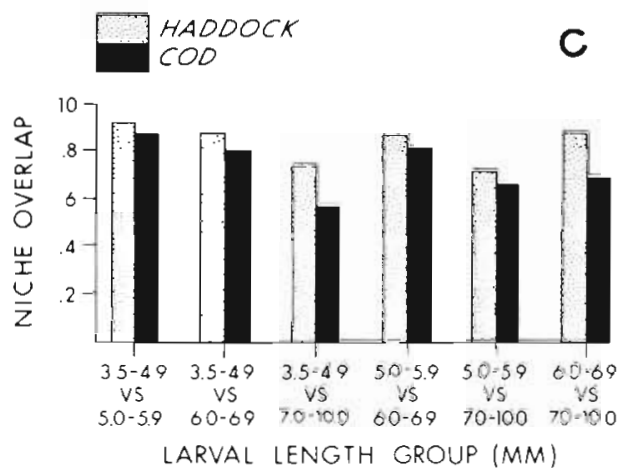

Fig. 8. Comparison of differences in the niche breadth index between different length categories of larvae (A). Comparison of dietary niche overlap indices between (B) and within (C) species over the range of length categories

\section{Niche breadth and overlap indices}

Dietary niche breadth indices for all larval length groups ranged from 0.23 to 0.32 (Fig. 8 A). Differences between species and length groups were negligible. The low values suggest that both species have a narrow feeding niche.

Dietary overlap indices were examined for between and within species differences among length groups. Values were high $(0.77$ to 0.88$)$ between species and did not change significantly among length groups (Fig. 8 B), indicating that interspecific competition for food is high between cod and haddock larvae. Index values fluctuated as expected among length groups within each species. Adjacent length groups had the highest dietary overlap while more distant pairings were lower because of size selective predation (Fig. $8 \mathrm{C}$ ). Values for cod larvae were lower than for haddock, suggesting that intraspecific competition is more severe among haddock larvae. The data also 
Table 6. Regression equations for standard length (SL) versus maxillary length (ML), skull width (SW) and eye height (EH). Between species differences in slopes were not significant $(0.02<\mathrm{P}<0.05$ )

\begin{tabular}{|cccc|}
\hline Haddock & & Cod \\
Equation & $r$ & Equation & $\mathrm{r}$ \\
\hline $\mathrm{ML}=-0.041+0.172 \mathrm{SL}$ & 0.922 & $\mathrm{ML}=-0.006+0.157 \mathrm{SL}$ & 0.942 \\
$\mathrm{SW}=0.120+0.107 \mathrm{SL}$ & 0.940 & $\mathrm{SW}=0.146+0.095 \mathrm{SL}$ & 0.957 \\
$\mathrm{EH}=0.021+0.095 \mathrm{SL}$ & 0.954 & $\mathrm{EH}=0.040+0.086 \mathrm{SL}$ & 0.925 \\
\hline
\end{tabular}

implies that intraspecific competition for available food among the smaller size groupings of larvae is greater than interspecific competition, reflecting the dietary differences between the young stages of larval cod and haddock.

\section{Morphoīogicaī measurements}

Linear regression equations were calculated for length versus skull width (representing mouth width), maxillary length, and eye height (Table 6). The slopes of the lines were compared between species by employing Students' t-test. No significant differences $(0.02<\mathrm{P}<0.05)$ were found. Apparently, neither species has a competitive advantage over the other due to differential exterior jaw or eye development.

\section{DISCUSSION}

In the 'critical period' of their early life history, young cod and haddock partition food resources, resulting in reduced interspecific competition. Cod are aggressive predators which feed on large prey items shortly after yolk sac absorption. Haddock are more passive foragers depending largely on less motile prey such as copepod eggs, phytoplankton, and copepod nauplii. After the larvae reach approximately $6.0 \mathrm{~mm}$, both species compete for the same food items. Presumably, larger larvae have greater food storage capabilities which render them less vulnerable to starvation (Hunter, 1976) and thus better adapted to share the same food resources. One might argue that the haddock larvae analysed here were weakened due to starvation, but both species appeared equally robust and the digestive tracts of young haddock were often filled with small prey. Thus, cod and haddock larvae can coexist together because they have adapted to rely on different sizes of prey for food shortly after yolk sac absorbtion.

There are no differences in external morphology that account for cod's greater success at capturing larger prey. They may possess more sophisticated sensory or locomotor apparatus, thus far undetected, but it is more likely that cod are simply more aggressive predators than haddock. Cod's utilization of a larger proportion of the prey assemblage earlier than haddock would seem to favor its survival. Clearly though, the benefits gained by feeding on larger prey may be offset by the energy expended in pursuit and capture. Conversely, since haddock are more passive predators, their basic metabolic requirements should be considerably lower. A larval cod less than $6.0 \mathrm{~mm}$ consumes more biomass than a corresponding haddock, but whether it contributes to growth or is expended in foraging is unknown.

The results of this study shed light on the outcome of the in vitro cod and haddock competition experiment conducted by Laurence et al. (1981) where 'survival was overwhelmingly in favor of cod'. Young larvae were placed in aquaria and fed varying concentrations of estuarine zooplankton composed chiefly of copepod nauplii, copepodites, and adults. Laurence et al. hypothesized that cod larvae were initially more successful than haddock at capturing the preferred prey organisms, which presumably were the same for both species because of what past field studies indicated. This study supports their contention that young cod are more accomplished predators, but it has also shown that younger larvae have different food preferences. Without their preferred food, it is understandable why haddock suffered almost total mortality competing with aggressive cod larvae for food resources not common in their natural environment.

Dietary niche breadth and overlap indices show both larvae utilize only a narrow portion of the prey assemblage and that there is intense competition between individuals of similar size for available food. However, many ecologists question whether overlap indices measure competition, and stress that they be interpreted with caution (e.g. Colwell and Futuyama, 1971; Abrams, 1980; Crecco and Blake, 1982). For example, 2 species can have completely overlapping diets but still not be in competition if resources are readily available (Hurlbert, 1978; Abrams, 1980). Since similar coexisting species have already co-evolved within the range of existing environmental variability, it seems unlikely that larval cod and haddock would still be 
competing for food resources. Dietary niche breadth and overlap indices are thus, at best, only indirectly related to the level of competition and would better serve as indicators of temporal changes in competition.

Previous studies of larval cod and haddock feeding on Georges Bank (Marak, 1960; Sherman et al., 1981) did not find evidence of resource partitioning among young larvae. Marak's findings are difficult to compare to the present analysis because all larvae were collected at the surface and thus had a different prey field to select from. Furthermore, Marak was unable to assess adequately differences between species because of low larval abundance. Sherman et al. compared feeding between cod and haddock larvae but partitioned their larvae into size groupings too large (4 mm increments) to distinguish differences in feeding between the species. It is also notable that the larvae examined by Sherman et al. lacked 2 food items often found in the diet of the larvae reported on herein: (1) the various life stages of Oithona similis, and (2) phytoplankton. The former may have been unavailable to the larvae, but the authors report that $O$. similis was a major constituent of the zooplankton community during the sampling period. This example of negative selectivity, coupled with evidence presented in this study strongly suggests that $O$. similis is very adept at avoiding capture by larval fish.

The smallest individuals of both species ingested significant amounts of phytoplankton, haddock more than cod. The nutritional contribution of phytoplankton to the larval diet is unknown, but there is growing evidence that certain species of phytoplankton may be a food source for the small stages of larvae. Ellertsen et al. (1980) have observed that young cod larvae in the laboratory actively feed on large phytoplankters at moderate densities. Lasker et al. (1970) successfully reared first feeding anchovy larvae from the Pacific with the dinoflagellate Gymnodinium splendens, and Scura and Jerde (1977) later showed that the survival of these larvae does not differ from those whose diet is supplemented with microzooplankton. Furthermore, Moffatt (1981) has observed the degradation of algal cell walls and intracellular chlorophyll in the intestines of anchovy larvae. This author and others have observed that armoured dinoflagellates are not digested by fish larvae, but other phytoplankters cannot be ignored as potential sources of nutrition for larval fish.

Coexistence of cod and haddock larvae on Georges Bank is not attributed to either absence of competition or character displacement. Neither principle exclusively applies, but each governs different periods of their early life histories. In the growth period where larval fish appear to be most susceptible to starvation, partitioning of resources minimizes interspecific com- petition. Later in life, when they are better suited to compete with one another, both utilize the same resources.

\section{LITERATURE CITED}

Abrams, P. (1980). Some comments on measuring niche overlap. Ecology 61 (1): 44-49

Blaxter, J. H. S. (1965). The feeding of herring larvae and their ecology in relation to feeding. Rep. Calif. Coop. Oceanic Fish. Invest. 10: 79-88

Berg, J. (1979). Discussion of methods of investigating the food of fishes with references to a preliminary study of the prey of Gobiusculus flavescens. Mar. Biol. 50: 263-273

Cohen, R. E., Lough, R. G. (1983). Prey field of larval herring, Clupea harengus on a continental shelf spawning area. Mar. Ecol. Prog. Ser. 10: 211-222

Colton, J. B., Green, J. R., Byron, R. R, Frisella, J. L. (1980). Bongo net retention rates as effected by towing speed and mesh size. Can. J. Fish. aquat. Sci. 37: 606-623

Colwell, R. K., Futuyma, D. J. (1971). On the measurement of niche breadth and overlap. Ecology 52: 567-576

Corkett, C. J., McLaren, I. A. (1978). The biology of Pseudocalanus. Adv. mar. Biol. 15: 1-231

Crecco, V., Blake, M. (1982). Feeding ecology and diet partitioning between larval American shad (Alosa sapidissima) and blueback herring (Alosa aestivalis) in the Connecticut River. Connecticut Dept. of Environmental Protection. (Unpubl. ms)

Ellertsen, B., Solemdal, P., Strømme, T., Tilseth, S., Westgård, T. (1980). Some biological aspects of cod larvae (Gadus morhua L.). FiskDir. Skr. Ser. HavUnders. 17: 29-47

Feinsinger, P., Spears, E., Poole, R. W. (1981). A simple measure of niche breadth. Ecology 62: 27-32

Gauld, D. T. (1966). The swimming and feeding of planktonic copepods. In: Barnes, H. (ed.) Some contemporary studies in marine science. George Allen and Unwin Ltd., London, p. 313-334

Hunter, J. R. (1976). Report of a colloquium on larval fish mortality studies and their relation to fishery research. NOAA Tech. Rep., NMFS Circ. 395: 1-5

Hunter, J. R. (1981). Feeding ecology and predation of marine fish larvae. In: Lasker, R. (ed.) Marine fish larvae. University of Washington Press, p. 34-77

Hurlbert, S. L. (1978). The measurement of niche overlap and some relatives. Ecology 59:67-77

Krebs, C. J. (1972). Ecology: the experimental analysis of distribution and abundance. Harper and Row, New York, p. 694

Lasker, R., Feder, H. M., Theilacker, G. H., May, R. C. (1970). Feeding, growth, and survival of Engraulis mordax larvae reared in the laboratory. Mar. Biol. 5: 345-353

Last, J. M. (1980). The food of twenty species of fish larvae in the west-central North Sea. Fish. Res. Tech. Rep. No. 60, Lowestoft, England, p. 1-44

Laurence, G. C. (1978). Comparative growth, respiration, and delayed feeding abilities of larval cod (Gaudus morhua) and haddock (Melanogrammus aeglefinus) as influenced by temperature during laboratory studies. Mar. Biol. 50: $1-7$

Laurence, G. C., Smigielski, A. S., Halavik, T. A., Burns, B. R. (1981). Implications of direct competition between larval cod (Gadus morhus) and haddock (Melanogrammus aeglefinus) in laboratory growth and survival studies at different food densities. Rapp. P.-v. Réun. Cons. int. Explor. Mer 178: 304-311 
Marak, R. R. (1960). Food habits of larval cod, haddock, and coalfish in the Gulf of Maine and Georges Bank area. J. Cons. int. Explor. Mer 25: 147-157

Marshall, S. M., Orr, A. P. (1955). The biology of a marine copepod. Oliver and Boyd, Edinburgh

Moffatt, N. M. (1981). Survival and growth of northern anchovy larvae on low zooplankton densities as affected by the presence of a Chorella bloom. Rapp. P.-v. Réun. Cons. int. Explor. Mer 178: 475-480

O'Brien, J. W. (1979). The predator-prey interaction of planktivorous fish and zooplankton. Am. Scient. 67: 572-581

Pearre, S. (1980). The copepod width-weight relation and its utility in food chain research. Can. J. Zool. 58: 1884-1891

Posgay, A., Marak, R. R. (1980). The MARMAP bongo zooplankton samplers. J. Northw. Atl. Fish. Sci. 1: 91-99

Saville, A. (1958). Mesh selection of plankton nets. J. Cons. Perm. Int. Explor. Mer 32: 232-248

Scura, E. D., Jerde, C. W. (1977). Various species of phyto- plankton as food for larval northern anchovy (Engraulis mordax) and relative nutritional value of the dinoflagellates (Gymnodinium splendens) and (Gonyaulax polydera). Fish. Bull. U. S. 75: 577-583

Sherman, K., Maurer, R., Byron, R., Green, J. (1981). Relationship between larval fish communities and zooplankton prey species in an offshore spawning ground. Rapp. P.-v. Réun. Cons. int. Explor. Mer 178: 289-294

Sheman, Kenneth (1980). MARMAP, a fisheries ecosystem study in the NW Atlantic: fluctuations in ichthyoplanktonzooplankton components and their potential for impact on the system. In: Diemer, F. P., Vernberg, F. J., Mirkes, D. Z., Belle, W. (ed.) Advanced concepts in ocean measurements for marine biology. Baruch Institute for Marine Biology and Coastal Research, University of South Carolina Press, p. 9-37

Shorigin, A. A. (1939). Food and food preference of some Gobiidae of the Caspian Sea. Zool. Zh. 18: 27-53

This paper was presented by Dr. K. Sherman; it was accepted for printing on October 3, 1983 